\title{
The effects of L-Dopa and $N$-(alpha-linolenoyl) tyrosine on 6-OH-DA lesions on dopamine level and activity, dyskinesia and homocysteinemia in rats
}

\author{
Shlomo Yehuda $^{1^{*}}$, Sharon Rabinovitz-Shenkar ${ }^{2}$ \\ ${ }_{1}^{1}$ Department of Psychology, Bar Ilan University, Ramat Gan, Israel; ${ }^{*}$ Corresponding Author: syehuda31@gmail.com \\ ${ }^{2}$ Department of Criminology, Bar Ilan University, Ramat Gan, Israel
}

Received 11 November 2012; revised 20 December 2012; accepted 29 December 2012

\begin{abstract}
The three major issues in L-DOPA therapy for Parkinson are: 1) rapid tolerance; 2) induced dyskinesia; 3) hyperhomocysteinemia. The newly synthesized molecule, which is an amide bond molecule composed of L-tyrosine and alphalinolenic acid, i.e., $\mathrm{N}$-(alpha-linolenoyl) tyrosine (NLT), represents a new class of molecules that combines essential amino acids with essential fatty acids. This study demonstrates that acute administration of NLT results in prolonged increase of the brain dopamine level, and that this molecule is able to overcome the three major problems associated with Parkinson's L-DOPA therapy: it is able to elevate brain dopamine levels and increase activity, without inducing tolerance, dyskinesia or hyperhomocysteinemia.
\end{abstract}

Keywords: Parkinson; Dopamine; Tolerance; Dyskinesia; Homocysteine; Rotational Behavior

\section{INTRODUCTION}

There is no ideal drug for treating Parkinson. The most understandable cure for Parkinson's should be a replace the dopamine in the brain that is being decreased during dopaminergic neuron degeneration. Dopamine itself, however, cannot pass the blood brain barrier to get into the brain. L-DOPA, the precursor of dopamine, can pass the blood brain barrier however. This means that we can administer L-DOPA, it will enter the brain, and then dopaminergic neurons with DOPA decarboxylase (the enzyme that breaks down L-DOPA) will convert LDOPA into dopamine, giving the dopaminergic neurons that remain in the brain more dopamine to release and make up for the ones that have died.

However, using L-DOPA therapy in treating Parkinson's disorder has some severe limitations, among them: 1) rapid tolerance to L-DOPA effects [1]; 2) L-DOPA induced dyskinesia [1];3) an elevated homocysteine level. Hyperhomocysteinemia is a risk factor of vascular disease, and potentially for dementia, diabetes, and depression [2].

A new class of bioactive molecules was recently created that combines an essential amino acid and an essential fatty acid, with an amide bond. The molecule travels intact in the blood and is able to cross into the brain. The naturally occurring enzymes (Fatty Acid Amide Hydrolase (FAAH)) are able to dissolve the amide bond and each of the components can act independently in the brain. The first example is the amide bond molecule, consisting of L-tyrosine and alpha-linolenic acid i.e., $N$-(alpha-linolenoyl) tyrosine (NLT). L-tyrosine is a precursor for dopamine; alpha-linolenic acid is a neuronal membrane stabilizer [3].

The possible protective role of essential fatty acids (EFA) in Parkinson has been described previously [4]. EFA are able to reduce the L-DOPA induced dyskinesia [5]. In addition, the significant inverse correlation between the homocysteine level and omega-3 fatty acids, and the possible protective effects of omega- 3 fatty acids against increased homocysteine concentrations has been previously proposed [6]. Therefore, the main open questions underlying this research were whether acute administration of NLT would significantly increase the level of brain dopamine and whether chronic administration of NLT (30 days) would result in lack of tolerance of dopaminergic effects, reduced dyskinesia and no increase in the homocysteine level.

\section{METHODS}

In the acute study, groups of adult SD (Sprague Dawley) rats $(150-200 \mathrm{~g})(n=56)$ were injected with 100 $\mathrm{mg} / \mathrm{kg}$ i.p. of NLT. The brains of 8 rats were removed and studied every hour for 6 hours, and the levels of dopamine (DA) and DOPAC were measured.

In the chronic study, groups of adult SD rats (150 $200 \mathrm{~g})(n=60)$ were subjected to left unilateral striatum 
6-OH-DA lesion. The method has previously been described (Yehuda, 2002). Only those rats whose lesion location and size were histological verified at the end of the experiment were included in this study. The Bar Ilan University Ethics Committee for Animal Experiments approved this study. The rats were challenged by chronic administration of $100 \mathrm{mg} / \mathrm{kg}$ NLT for 30 days. As a control, other groups of rats $(n=12$ in each experimental group) were injected with either $1 \mathrm{cc}$ Saline $(0.9 \% \mathrm{NaCl})$, $100 \mathrm{mg} / \mathrm{kg}$ L-DOPA, $100 \mathrm{mg} / \mathrm{kg}$ alpha linolenic acid, or $5 \mathrm{mg} / \mathrm{kg}$ d-amphetamine. The control groups were treated with the gold standard of anti-Parkinson drugs, e.g., LDOPA, another powerful dopaminergic agent, e.g., damphetamine, and also with alpha linolenic acid. All injecttions were i.p.. The rats were treated for 30 days.

\subsection{Catecholamine Analysis}

Brains were dissected quickly on an ice-chilled surface using a "brain block" and stored in microcentrifuge tubes at $-80^{\circ} \mathrm{C}$ until analysis. Cold mobile phase and internal standard (isoproterenol) were added to the samples just prior to analysis. Samples were vortexed and sonicated (Branson Cell Disruptor) to release catecholamines, and then centrifuged at $14,000 \times \mathrm{g}$ for $18 \mathrm{~min}$. A $200-\mu \mathrm{l}$ aliquot of the supernatant was placed in an autosampler tube for injection onto the column. Dopamine (DA), and selected metabolites, 3,4-dihydroxyphenylacetic acid (DOPAC) and homovanillic acid (HVA), were quantified using reverse-phase ion pair chromotography with electrochemical detection. A Metachem Technologies (Metasil) reversed-phase column $(4.6 \mathrm{~mm}$ inner diameter $\times 100$ $\mathrm{mm}$ long) was used in the separation. The mobile phase consisted of $0.05 \mathrm{M}$ sodium phosphate, $0.03 \mathrm{M}$ citric acid, $0.1 \mathrm{mM}$ EDTA, $2 \mathrm{mM}$ sodium octyl sulfate, and 14\% $18 \%$ HPLC-grade methanol at $\mathrm{pH} 3.4$. The flow rate was $0.8 \mathrm{ml} / \mathrm{min}$.

Neurotransmitters were detected with a glossy carbon electrode set at $+0.65 \mathrm{~V}$. The lower limit of $20 \mathrm{fmol}$ of DA in a $20 \mu \mathrm{l}$ sample was consistently detected. Concentrations of DA, DOPAC, and HVA, in striatal tissue, were calculated by linear regression based on a standard curve generated using a PE Nelson 500 series Interface and appropriate software.

\subsection{Lesions}

All surgery was performed under sodium pentobarbital anesthesia (pentobarbital sodium, $50 \mathrm{mg} / \mathrm{ml}$ as required). The rats were pretreated with $10 \mathrm{mg} / \mathrm{kg}$ i.p. of desimipramine (in order to prevent damage to the noradrenergic neuron) and fixed in a Kopf stereotaxic instrument (Model 900). Left unilateral 6-OH-DA (12 $\mu \mathrm{g}$ in $4 \mu \mathrm{l}$ of saline containing $0.05 \%$ ascorbic acid) lesions were made. In order to verify the location of the injection, the site was marked by electrical current, which was deli- vered by the needle of the injection. The electrical current was constant $(0.5 \mathrm{mV}$ for $1 \mathrm{~s})$ and was passed through a stainless-steel insulated needle, bared only in the tip. The coordinates (modified from Konig and Klippel, 1963 [7]) for the caudate nucleus lesion were A 8.5, L 2.2, and $\mathrm{B}+1.8$ from the interaural line. The chemical lesion was made in the left side of the brain. After surgery, each rat was placed in an individual cage.

\subsection{Rotational Behavior}

Rotational behavior was tested in a rotometer $30 \mathrm{~min}$ after injection for $30 \mathrm{~min}$ (i.e., between 30 and $60 \mathrm{~min}$ after injection), with each rat mounted in a special harness and placed in an acrylic, transparent dome of $41 \mathrm{~cm}$ radius. Rotational movements were transuded from the harness via a stainless-steel tube (1/8 in.) and a precision universal joint (Pic BC 12) to a 5-K linear potentiometer (Spectrol), which received an excitation current from Sanborn polygraph. The potentiometer (preamplifier $8805 \mathrm{~A}$ ) measured and recorded the changes in the amount of current resulting from the rotational movements. A turn to the right deflected the recording pen upward, while a leftward turn deflected the pen downward. Continuous recordings were made at a speed of 1 $\mathrm{mm} / \mathrm{s}$.

\subsection{AIMs}

AIMs score ratings were performed by an investigator, who was kept unaware of the pharmacological treatment administered to each rat (experimentally blinded). In order to quantify the severity of the AIMs, rats were observed individually in their standard cages every $20 \mathrm{~min}-$ utes, 20 - 180 minutes after injection of the drug [8]. The rats' AIMs were classified into four subtypes: 1) axial AIMs, i.e., dystonic or choreiform torsion of the trunk and neck towards the side contralateral to the lesion; 2) limb AIMs, i.e., jerky and/or dystonic movements of the forelimb contralateral to the lesion; 3) orolingual AIMs, i.e., twitching of orofacial muscles, and bursts of empty masticatory movements with protrusion of the tongue towards the side contralateral to the lesion. Each of the three subtypes was scored on a severity scale from 0 to 4 , were $0=$ absent, $1=$ present during less than half of the observation time, $2=$ present for more than half of the observation time, 3 = present all the time but suppressible by external stimuli, and $4=$ present all the time and not suppressible by external stimuli. Thereafter, scores from these three AIM subtypes were summed (maximum score was 12 points).

\subsection{Homocysteine Level}

Blood samples were drawn from the tail, and promptly 
centrifuged at $1750 \times \mathrm{g}$ for $15 \mathrm{~min}$ at $4^{\circ} \mathrm{C}$. The plasma was stored frozen at $-70^{\circ} \mathrm{C}$. Plasma homocysteine was analyzed by the HPLC (Waters 474, Milford, MA) fluorescence detection method developed by Araki and Sako [9] and Zesiewicz [10].

\subsection{Statistical Methods}

The data are expressed as mean \pm standard deviation (SD). The Student's $t$-test, Chi-square test and multivariate analysis of variance (MANOVA) were used to evaluate the significance of differences between means.

\section{RESULTS}

The results of this study showed the following:

1) Administration of NLT results in prolonged increase of the brain DA level. The brain DA level increases $400 \%$ from time $0(85.5 \pm 38.1 \mathrm{pmol} / \mathrm{g}$ of brain $)$ to time 6 hours $(448.7 \pm 65.4)$ (Student's $t$-test, $t=11.78, d f=14, p \leq$ $0.001)$. The levels of DOPAC were not statistically significantly changed (Table $\mathbf{1}$ ).

2) While L-DOPA and d-amphetamine induce tolerance, no tolerance was observed regarding the NLT effects in the Rotational Behavior model, which served as a screening tool for potential dopaminergic anti-Parkinson drugs [4] $\left(\chi^{2}=15.06, d f=4, p \leq 0.003\right)$ (Table 2). Only the NLT treated group showed significant ipsilatral rotational behavior.

3) While L-DOPA induced significant dyskinesia, measured as Abnormal Involuntary Movements (AIMs) (Dekundy et al. 2007), NLT treatment did not induce AIMs (MANOVA, $F(4,55)=15.12, p \leq 0.001$, and Duncan's post hoc $t$-test) (Table 3). An increase in AIMs score was noted in the L-Dopa treated group on day 12, and the AIMs scores were significantly increased thereafter.

4) Chronic administration of NLT does not induce an

Table 1. Brain DA and DOPAC levels after acute treatment of NLT.

\begin{tabular}{ccc}
\hline & DA & DOPAC \\
\hline 0 & $85.5 \pm 38.1$ & $663 \pm 120.0$ \\
1 & $144.8 \pm 24.5$ & $748.5 \pm 98.3$ \\
2 & $235.4 \pm 38.8$ & $760.1 \pm 125.4$ \\
3 & $299.5 \pm 58.8$ & $785.7 \pm 141.5$ \\
4 & $320.2 \pm 40.6$ & $749.2 \pm 133.3$ \\
5 & $380.4 \pm 50.5$ & $710.9 \pm 142.3$ \\
6 & $448.7 \pm 65.4$ & $666.8 \pm 100.5$ \\
\hline
\end{tabular}

Effects of acute treatment of NLT on brain DA and DOPAC $(\mathrm{pmol} / \mathrm{g}$ of brain). Cell entries represent the levels $(M \pm S D), 6$ hours after treatment. See text for significant statistical differences between the experimental groups. There were 8 rats per group.
Table 2. Ipsilateral turns in 6-OH-DA-lesioned rats.

\begin{tabular}{ccccccc}
\hline & \multicolumn{3}{c}{ Day 0 } & \multicolumn{4}{c}{ Day 30 } \\
\cline { 2 - 7 } & $\mathrm{L}$ & $\mathrm{R}$ & $\% \mathrm{~L}$ & $\mathrm{~L}$ & $\mathrm{R}$ & $\% \mathrm{~L}$ \\
\hline Saline & $4 \pm 0$ & $26 \pm 2$ & 13 & $5 \pm 0.5$ & $30 \pm 2$ & 14 \\
d-Amphetamine & $78 \pm 7$ & $4 \pm 0$ & 95 & $40 \pm 4.5$ & $20 \pm 3.5$ & 58 \\
L-Dopa & $255 \pm 1.5$ & $8 \pm 2$ & 86 & $25 \pm 3$ & $15 \pm 2.5$ & 60 \\
Alpha-Linolenic & $8 \pm 1$ & $32 \pm 4$ & 20 & $10 \pm 1$ & $30 \pm 2$ & 25 \\
NLT & $98 \pm 10$ & $2 \pm 0$ & 98 & $72 \pm 4$ & $8 \pm 2$ & 90 \\
\hline
\end{tabular}

L, Left; R, Right. Rotational behavior was measured as described in the text (Yehuda, 2002). Cell entries represent the number and direction of rats' turns during the measurement period, for the separate experimental groups $(\mathrm{M} \pm \mathrm{SD})$, on day 0 and day 30 . See text for significant statistical differences between the experimental groups. There were 12 rats per group.

Table 3. Development of AIMs in rats treated for 30 days.

\begin{tabular}{cccccc}
\hline Day & Saline & d-Amphetamine & L-Dopa & Linolenic & NLT \\
\hline 0 & $0.2 \pm 0.1$ & $7.2 \pm 1.0$ & $0.2 \pm 0.1$ & $0.4 \pm 0.4$ & $0.5 \pm 0.2$ \\
6 & $0.2 \pm 0.1$ & $4.0 \pm 0.9$ & $0.3 \pm 0.1$ & $0.5 \pm 0.4$ & $0.4 \pm 0.3$ \\
9 & $0.2 \pm 0.1$ & $1.5 \pm 1.0$ & $0.6 \pm 0.5$ & $0.6 \pm 0.3$ & $0.5 \pm 0.3$ \\
12 & $0.2 \pm 0.2$ & $0.5 \pm 0.4$ & $1.3 \pm 1.0$ & $0.3 \pm 0.2$ & $0.4 \pm 0.2$ \\
15 & $0.2 \pm 0.1$ & $0.3 \pm 0.3$ & $6.0 \pm 1.0$ & $0.6 \pm 0.4$ & $0.5 \pm 0.4$ \\
30 & $0.2 \pm 0.1$ & $0.4 \pm 0.3$ & $8.5 \pm 2.7$ & $0.7 \pm 0.4$ & $0.6 \pm 0.3$ \\
\hline
\end{tabular}

AIMs were measured as described in the text. Cell entries represent the number and direction of rats' turns during the measurement period, for the separate experimental groups $(\mathrm{M} \pm \mathrm{SD})$, on day $0,6,9,12,15$, and 30 . See text for significant statistical differences between the experimental groups. There were 12 rats per group.

elevation in plasma homocysteine levels (MANOVA, $F(4,55)=13.87, p \leq 0.001$, and Duncan's post hoc $t$-test) (Table 4). The L-Dopa treated group exhibited a significant increase in homocysteine levels on day 9, and the homocysteine level was even higher on day 30 . No such increase was found in any other experimental group.

\section{DISCUSSION}

It is important to note that: 1) acute administration of NLT resulted in prolonged increase of the DA level (400\%) during the first 6 hours after injection, without increase in the DOPAC level. This finding indicates that DA is able to accumulate in the axons terminals without metabolites by DOPAC inside the axons; 2) acute administration of d-amphetamine, L-DOPA, and NLT induced significant rotational behavior toward the lesion side, but saline and alpha linolenic did not. In contrast, in chronic administration, only the NLT group did not exhibit tolerance; 3) only acute administration of d-amphetamine induced AIMs, while chronic administration of L-DOPA induced AIMs, but not d-amphetamine or 
Table 4. Development of hyperhomocysteinemia.

\begin{tabular}{|c|c|c|c|c|c|}
\hline Day & Saline & D-Amphetamine & L-Dopa & Linolenic & NLT \\
\hline 0 & $6.20 \pm 1.5$ & $6.20 \pm 1.0$ & $6.2 \pm 1.0$ & $6.2 \pm 1.0$ & $6.2 \pm 1.0$ \\
\hline 6 & $6.25 \pm 1.0$ & $6.4 \pm 1.3$ & $6.4 \pm 1.4$ & $7 \pm 1.5$ & $6.5 \pm 1.3$ \\
\hline 9 & $6.5 \pm 1.5$ & $6.5 \pm 1.4$ & $9.8 \pm 1.9$ & $6.9 \pm 2.0$ & $7.0 \pm 1.9$ \\
\hline 12 & $6.10 \pm 1.5$ & $6.7 \pm 1.4$ & $14.9 \pm 1.5$ & $7.4 \pm 2.2$ & $6.70 \pm 1.8$ \\
\hline 15 & $6.25 \pm 1.5$ & $7 \pm 1.5$ & $18.2 \pm 2.5$ & $7.0 \pm 1.7$ & $5.8 \pm 1.7$ \\
\hline 30 & $6.25 \pm 1.5$ & $7.2 \pm 1.5$ & $22.0 \pm 3.0$ & $6.4 \pm 1.1$ & $5.5 \pm 1.0$ \\
\hline
\end{tabular}

The plasma level of homocysteine was measured in blood obtained from the tail vein. HPLC method was used as previously described [11]. Cell entries represent the homocysteine level expressed in micromole/ $\mathrm{L}(\mathrm{M} \pm \mathrm{SD})$, on day $0,3,6,9,12,15$, and day 30 . See text for significant statistical differences between the experimental groups. There were 12 rats per group.

NLT; 4) only chronic administration of L-DOPA resulted in an elevation of the homocysteine level. NLT, which contains tyrosine, which is converted to L-DOPA, did not induce an elevation of the homocysteine level.

It seems that NLT does not produce tolerance due to the effect of the essential fatty acid. It has been shown that the L-DOPA induced hyperhomocysteinemia is mediated by catechol-O-methyltransferase (COMT) activity. COMT inhibitors have been recommended as add-on medications to L-DOPA treatment, however, with limited success [10]. The inhibition of the hyperhomocysteinemia in NLT treatment can be explained by the fact that tyrosine is not a substrate for COMT, or by the fact that the COMT is inhibited by alpha-linolenic acid. More studies are needed to clarify the mode of action.

\section{ACKNOWLEDGEMENTS}

We would like to thank Diascience Corp. (New York, N.Y. USA) for their generous financial support.

\section{REFERENCES}

[1] Nutt, J.G., Obeso, J.A. and Stocchi F. (2000) Continuous dopamine-receptor stimulation in advanced Parkinson's disease. Trends in Neurosciences, 10, S109-S15. doi:10.1016/S1471-1931(00)00029-X
[2] Troen, A. and Rosenberg, I. (2005). Homocysteine and cognitive function. Seminars in Vascular Medicine, 2, 209214. doi: $10.1055 / \mathrm{s}-2005-872406$

[3] Yehuda, S., Rabinovitz, S. and Mostofsky, D.I. (2005) Essential fatty acids and the brain: from infancy to aging. Neurobiology of Aging, 26, 98-102. doi:10.1016/j.neurobiolaging.2005.09.013

[4] Yehuda, S. (2002) Possible anti-Parkinson properties of N-(alpha-linolenoyl) tyrosine: a new molecule. Pharmacology, Biochemistry and Behavior, 72, 7-11. doi:10.1016/S0091-3057(01)00646-3

[5] Samadi, P., Gregiore, L., Rouillard, C., Bedard, P.J., Di Paolo, T. and Levesque, D. (2006) Docosahexaenoic acid reduces levadopa-induced dyskinesias in 1-methyl-4-phenyl-1,2,3,6-tetrahydropyridine monkeys. Annals of Neurology, 59, 282-288. doi:10.1002/ana.20738

[6] De Bree, A., Mennen, L.I., Hercberg, S. and Galan, P. (2004) Evidence for a protective (synergistic?) effect of B-vitamins and omega-3 fatty acids on cardiovascular diseases. European Journal of Clinical Nutrition, 58, 732744. doi:10.1038/sj.ejen.1601871

[7] Konig, J.F.R. and Klippel, R.A. (1963) The rat brain: a stereotaxic atlas of the forebrain and lower parts of the brain stem. Williams and Wilkins, Baltimore.

[8] Dekundy, A., Lundblad, M., Danysz, W. and Cenci, M.A. (2007) Modulation of L-DOPA-induced abnormal involuntary movements by clinically tested compounds: further validation of the rat dyskinesia model. Behavioural Brain Research, 179, 76-89. doi:10.1016/j.bbr.2007.01.013

[9] Araki, A. and Sako, Y. (1987) Determination of free and total homocysteine in human plasma by high-performance liquid chromatography with fluorescence detection. Journal of Chromatography, 422, 43-52.

[10] Zesiewicz, T.A., Wecker, L., Sullivan, K.L., Merlin, L.R. and Hauser, R.A. (2006) Topiramate-induced psychosis in patients with essential tremor: Report of 2 cases. Clinical Neuropharmacology, 29, 106-111. doi:10.1097/01.WNF.0000220817.94102.95

[11] Kim, J.M., Lee, H. and Chang, N. (2002) Hyperhomocysteinemia due to short-term folate deprivation is related to electron microscopic changes in the rat brain. Journal of Nutrition, 132, 3418-3421. 